Volume: 2, Issue: 4, December 2017, Pages: 219-222, DOI: http://dx.doi.org/10.19082/ah219

\title{
INOPERABLE DISSEMINATED PULMONARY AND PLEURAL CYSTIC HYDATIDOSIS PRESENTING WITH AN OPAQUE HEMITHORAX IN A 14-YEAR-OLD CHILD
}

\author{
Firas Alakhras Aljanadi
}

M.D., MSc., Damascus University Cardiac Surgery Hospital, Damascus University, Damascus, Syria

\section{TYPE OF ARTICLE: CASE REPORT}

\begin{abstract}
Introduction: Hydatidosis is a parasitic infection caused by Echinococcus granulosus. People can be infected when they get worm eggs from contaminated puppies during their childhood or from sullied uncooked vegetables in sheep-raising zones of the world. Hydatid disease is generally phenomenal in children, and advanced stages of hydatidosis that are inoperable are uncommon and rarely described in the medical literature.

Case presentation: We report a case of 14-year-old child diagnosed with advanced inoperable disseminated multiple hydatid cysts who presented with shortness of breath and left shoulder pain. The chest x-ray revealed a complete opacification of the left hemithorax with deviation of the trachea to the right. Thoracic computed tomography scan showed the left hemithorax occupied almost completely with multiple pulmonary, pleural, and chest wall cysts in variable sizes and sites. Abdominal ultrasound showed hepatic cysts. Serology test was positive for hydatid cyst and aspirated fluid showed well-defined scolices. Unfortunately, because of multiorgan disease and multiple cysts, surgery was abandoned at this stage, and medical treatment was started with follow-up schedule.

Conclusion: In endemic areas, hydatid disease is possible among children, and having a high index of suspicion of hydatid disease is necessary. It should be among the differential diagnoses of any cystic mass lesion. Advanced disseminated disease can be seen in children and can present with generalized left chest opacity. While surgery remains the primary choice of treatment, medical treatment may be preferred in disseminated and multi-organ disease with follow-up evaluations.
\end{abstract}

KEYWORDS: Hydatid cyst, Cystic echinococcosis, Echinococcus granulosus, Pulmonary, Hydatidosis

\section{INTRODUCTION}

The pathogen of echinococcosis or hydatid disease is the larvae of the tapeworm Echinococcus (1). In cystic echinococcosis, humans are considered as an accidental host and are usually infected by dealing with infected dogs (1). The most frequent affected organs are the liver and lungs (1). Unlike an adult, lung involvement is more common than liver involvement in children, with frequencies of $64 \%$ and $28 \%$, respectively (2). Both organs, the liver and lung, are involved simultaneously in around $5 \%-13 \%$ of cases (3). Isolated pulmonary cysts are more common in children (2). In straightforward cases of cystic echinococcosis, where cysts number and organ involvement is limited, cysts are not in risky sites, the infection is not very advanced, and most well-known type of treatment is surgical removal of the cysts consolidated with chemotherapy utilizing Albendazol and/or Mebendazole before and after surgery (4). However, in any case, if there are cysts in various organs or tissues, or they are in risky locations, or in high-surgical-risk patients, surgery ends up plainly unrealistic and impractical; and for such inoperable cases, chemotherapy and/or PAIR (puncture-aspiration-injection-respiration) might be considered as the alternative options of treatment (4).

Few cases about disseminated multiorgan hydatidosis in children were reported; however, presenting as a total opaque hemithorax, primarily due to an advanced hydatidosis, was not reported previously in the medical literature. We present here a noteworthy rare case of advanced multiple organ hydatid disease affecting the left lung, pleura, pleural cavity, chest wall, and liver presenting as a total opaque left hemithorax in a 14-year-old child, where surgery was not the first choice and medical treatment was indicated.

\section{Correspondence:}

Dr. Firas Alakhras Aljanadi, Damascus University Cardiac Surgery Hospital, Damascus University, Damascus, Syria. Tel: +44.7405163591, Email: firasaljanadi@gmail.com

Received: February 20, 2017, Accepted: April 16, 2017, Published: December 2017

iThenticate screening: February 23, 2017, English editing: April 29, 2017, Quality control: May 20, 2017

(C) 2016 The Authors. This is an open access article under the terms of the Creative Commons Attribution-NonCommercialNoDerivs License, which permits use and distribution in any medium, provided the original work is properly cited, the use is non-commercial and no modifications or adaptations are made. 


\section{CASE PRESENTATION}

\section{1. Clinical presentation}

A 14-year-old male child presented to a local medical center with an attack of shortness of breath and left shoulder pain radiating over the left arm. This was associated with dry cough and two episodes of haemoptysis about $100 \mathrm{ml}$ each time. There was no fever, anorexia, or weight loss. There was no significant previous medical history except a chronic dry cough over the last two years. After chest x-ray (Figure 1.A), which showed a left opaque hemithorax, absent left-lung markings, and mild tracheal shift to the right, he was referred to the hospital for suggested chest drain insertion and further investigations.

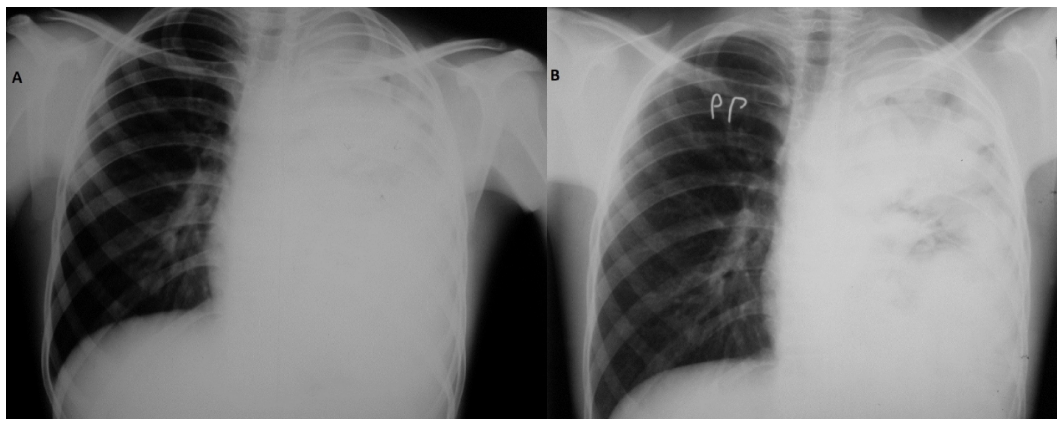

Figure 1. Chest x-ray. A: A posterior-anterior view shows opacity of left hemithorax, absent left-lung markings, and mild tracheal shift to the right. B: Initial chest $\mathrm{x}$-ray on admission. Homogenous left-sided opacity with small patchy aired areas and tracheal shift to the right.

\section{2. Physical examination}

On examination, he was haemodynamically stable with respiratory rate $26 / \mathrm{min}$. It was noticed that the trachea was shifted to the right with limitation in the left chest movements associated with left dullness and diminished breath sounds without wheezing or stridor. The cardiovascular examination was unremarkable and abdominal examination revealed no organomegaly.

\section{3. Laboratory and imaging findings}

Blood tests showed 7\% eosinophilia, the erythrocyte sedimentation rate (ESR) $60 \mathrm{~mm} / \mathrm{hrs}$. And the arterial blood gases (ABG) showed O2 saturation 95\%. Chest x-ray on admission (Figure 1.B) showed homogenous left-sided opacity with small patchy aired areas lateral to the left hilum, tracheal shift to the right and compensatory hyperinflation of the right hemithorax. Chest computed-tomography (CT) scan (Figures 2, 3, 4) showed disseminated multiple cysts with variable sizes and distinguished walls with clear fluid content occupying most of the left chest, in the lung, pleura, pleural cavity, and chest wall with small remaining aired pulmonary parenchyma. Anti-echinococcus antibodies test was done and found significantly positive at 1:1200. Additionally, examination of fluid carefully aspirated under ultrasound (US) guidance showed well-defined scolices. Abdominal ultrasound revealed a cystic lesion on the inferior hepatic edge measures $20 \mathrm{~mm} \times 16 \mathrm{~mm}$, most probably a young hydatid cyst.
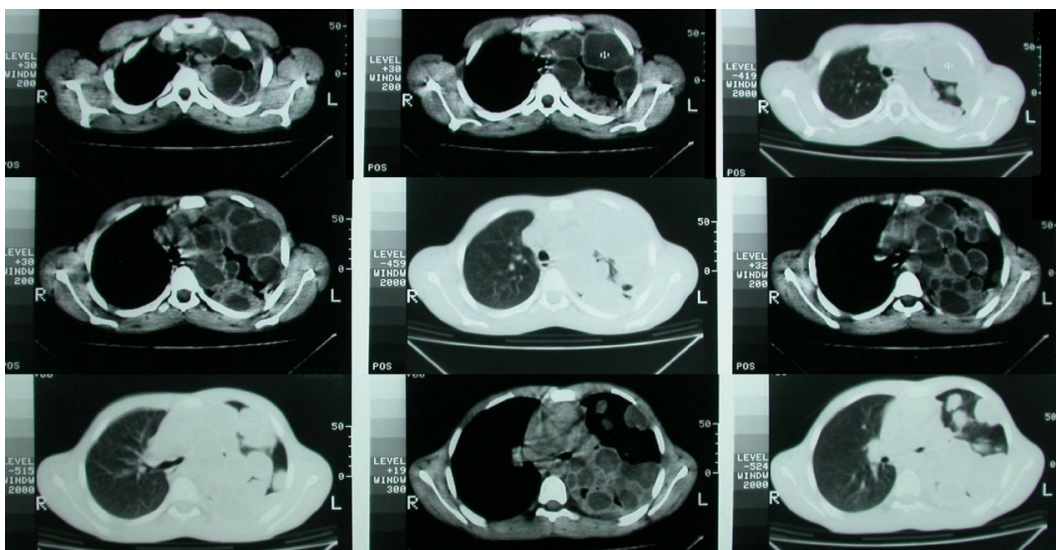

Figure 2. Chest computed tomography (CT) images show multiple hydatid cysts located in the left thorax as welldefined cystic lesions with variable sizes. 

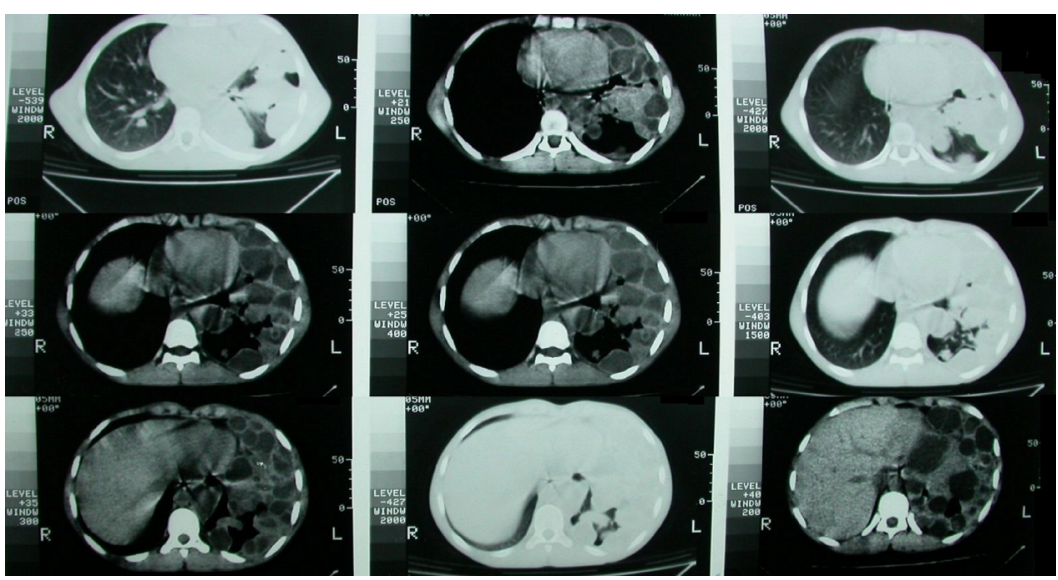

Figure 3. Computed tomography (CT) scan shows multiple hydatid cysts located in the left thorax as well-defined cystic lesions with different sizes.

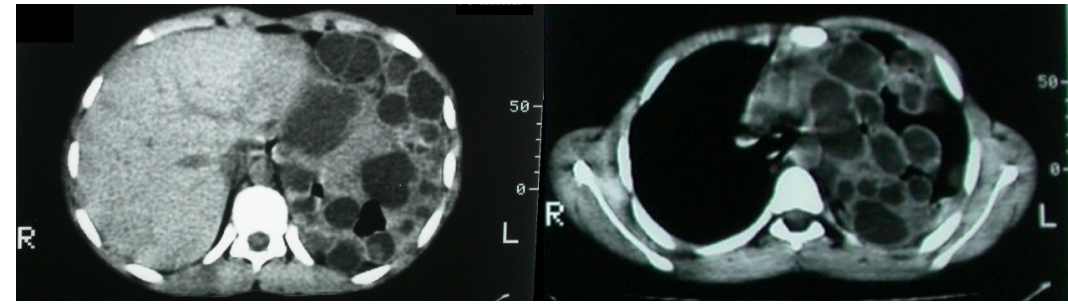

Figure 4. Computed tomography (CT) scan images, disseminated hydatidosis fill the left thorax.

\section{4. Treatment and follow-up}

According to these results, surgery was not indicated at that moment and was not done. The patient was discharged on Albendazol and Praziquantel for six months to follow-up afterward for reevaluation. After six months of medical treatment, there was a significant improvement in symptoms and chest x-ray. Follow-up on six-month-interval basis was considered.

\section{DISCUSSION}

Hydatid disease is a parasitic disease that is endemic in many parts of the world (3). Hydatid disease can occur almost anywhere in the body and demonstrates a variety of imaging features that vary according to growth stage, associated complications, and affected tissue (3). Though hydatid disease is widely endemic in Middle Eastern countries $(5,6)$, in children; the advanced disseminated disease is rarely seen nowadays, and the involvement of multiple sites in young children with enormous cysts has been very rarely reported in the medical literature (3, 7). Though it was previously reported that a massive pleural effusion might follow a ruptured hydatid cyst (8), however, finding a total left opaque hemithorax as a result of disseminated hydatid cysts invading the whole hemithorax and showing the clinical presentation as a massive pleural effusion, as in this case, was never previously reported. The background of the child, as living in a sheep rearing area, and the investigations could help us to reach the diagnosis. The final diagnosis was multiple disseminated pleural, chest wall, and pulmonary hydatidosis associated with hepatic hydatid disease. What was remarkable also is the advanced stage of this disease in a child where cysts invaded most of the thorax and scattered in the left pleural cavity.

As the polycystic echinococcosis is not well described or found in many individuals, the treatment of polycystic echinococcosis is less well-defined than that of cystic and alveolar echinococcosis. Surgical removal of cysts was the best treatment for the past two sorts of echinococcosis, whereas medicinal treatment is the advised management approach for polycystic echinococcosis (4). Medical therapy is indicated in patients with contraindication for surgery, with poor surgical risk, refusal for surgery, multi-organ disease, or multiple cysts (2). Unfortunately, due to multiorgan disease and multiple cysts, surgery was considered contraindicated in our case. Dispersed hydatid disease is an absolute indication for antihelminthic drug therapy, and medicinal treatment is by all accounts more viable for pulmonary hydatidosis (3). However, hydatid cysts with sizes surpassing $6 \mathrm{~cm}$ in diameter should not be dealt with medically (3), and in our patient the sizes of all cysts were under $6 \mathrm{~cm}$. Lately, trials with Mebendazole or 
Albendazol have given encouraging results, particularly in instances of multiple or inoperable cysts (7). Albendazol $(10 \mathrm{mg} / \mathrm{kg})$ is the most usually utilized medication, given as a 28 -day cycle with a two-week break between cycles (3). While Albendazol is the favored medication, Mebendazole can likewise be utilized if the treatment is for a prolonged period of time $(3,4)$; it has been claimed that long-term medical therapy with Mebendazole can be lifesaving in cases of cystic echinococcosis that could not be managed by surgery (3). Some authors revealed that Mebendazole treatment at a dosage of $40 \mathrm{mg} / \mathrm{kg}$ every day delivered a significant effect within the first month of treatment; and the whole lung cysts, the singular cyst in the kidney, and the majority of the smaller cysts in the liver vanished within 13 months (7). Others found that medical treatment with Benzimidazoles (Mebendazole or Albendazol) is profitable in scattered disseminated disease, including secondary pulmonary or pleural hydatidosis, poor surgical risk patients, and when there is intraoperative spillage of hydatid cysts fluid $(1,5)$. However, assessing and evaluating the accomplishment of treatment is not easy and often requires regular follow-up and imaging (1) and when medical therapy fails surgery is advised (4). Depending on these published results, we discharged our patient on a combination of Albendazol and Praziquantel to be followed up every six months and managed according to the findings afterward.

\section{CONCLUSIONS}

In conclusion, in endemic areas, hydatid disease is possible among children, and having a high index of suspicion of hydatid disease is necessary and should be among the differential diagnoses of any cystic mass lesion. History, clinical, and epidemiological information in addition to serology tests, CT scan, and ultrasound help to reach the diagnosis. Advanced disseminated disease can be seen in children and can present with generalized left chest opacity. While surgery remain the primary choice of treatment; however, medical treatment may be preferred in disseminated and multiple disease with follow-up evaluations.

\section{ACKNOWLEDGMENTS:}

I would like to thank the Thoracic Surgery Department and the surgical, anaesthetic, and theatre staff at AlMouwasat University Hospital for their cooperation and forbearance.

\section{CONFLICT OF INTEREST:}

The authors declare that they have no conflict of interests.

\section{REFERENCES:}

1) Morar R, Feldman C. Pulmonary echinococcosis. European Respiratory Journal. 2003 Jun 1; 21(6): 106977. doi: 10.1183/09031936.03.00108403.

2) Sarkar M, Pathania R, Jhobta A, Thakur BR, Chopra R. Cystic pulmonary hydatidosis. Lung India: official organ of Indian Chest Society. 2016 Mar; 33(2): 179. doi: 10.4103/0970-2113.177449.

3) Harjai MM, Saxena S, Devgan A, Kakkar S. Disseminated Hydatid Disease in a Child. European journal of pediatric surgery. 2009 Aug; 19(04): 270-1. doi: 10.1055/s-2008-1039201.

4) Eckert J, Deplazes P. Biological, epidemiological, and clinical aspects of echinococcosis, a zoonosis of increasing concern. Clinical microbiology reviews. 2004 Jan 1; 17(1): 107-35. doi: 10.1128/CMR.17.1.107-135.2004.

5) Çelik M, Senol C, Keles M, Halezeroglu S, Urek S, Haciibrahimoglu G, Ersev AA, Arman B. Surgical treatment of pulmonary hydatid disease in children: report of 122 cases. Journal of pediatric surgery. 2000 Dec 31; 35(12): 1710-3. doi: 10.1053/jpsu.2000.19219.

6) Gupta R, Sharma SB, Prabhakar G, Mathur P. Hydatid disease in children: Our experience. Formosan Journal of Surgery. 2014 Dec 31; 47(6): 211-20. doi: 10.1016/j.fjs.2014.12.001.

7) Kaya Z, Gursel T. A pediatric case of disseminated cystic echinococcosis successfully treated with mebendazole. Japanese journal of infectious diseases. 2004 Feb 1; 57(1): 7-9. PMID: 14985629. PMid: 14985629.

8) Chakrabarti S, Patra A, Biswas P, Mandal K. Pulmonary hydatid cyst presenting as massive unilateral pleural effusion. The Journal of Association of Chest Physicians. 2015 Jan 1; 3(1): 20. doi: 10.4103/23208775.146847. 